Results Two groups did not differ significantly in infant and mother demographic information. After one week of intervention, the mean difference of total crying time, duration and severity were 4.08 (1.83) time/day, 2.81 (1.77) hour/day and 2.9 (2.37) in massage group and 0.56 (2.28) time/day, 0.27 (1.09) hour/day and 0.02 (1.64) in vibrating group, respectively. The mean of total crying time, duration and severity decreased in both groups but there was a more significant reduction in crying times, duration and severity in massage group than the rocking group.

Conclusions Our findings demonstrated that infant massage was effective in reducing the time, duration, and severity of crying in colicky infants.

\section{PAIN IN CHILDREN: KNOWLEDGE AND PERCEPTIONS OF NURSING STAFF AT A RURAL TERTIARY CARE TEACHING HOSPITAL IN WESTERN INDIA}

doi:10.1136/archdischild-2012-302724.1625

${ }^{1} \mathrm{AS}$ Nimbalkar, ${ }^{1} \mathrm{AR}$ Dongara, ${ }^{2} \mathrm{JD}$ Ganjiwale, ${ }^{1,2}$ SM Nimbalkar. 'Department of Pediatrics, Pramukhswami Medical College; ${ }^{2}$ Central Research Services, Charutar Arogya Mandal, Anand, India

Backgrounds and Aims Pain is a common cause for which patients seek treatment and an unpleasant side effect of our treatment. Young children are not able to express their pain. It is the care-givers responsibility to assess and manage their pain. Caregivers perception of the child's pain depends on various factors. We studied the perception and knowledge regarding pain amongst nursing staff at our centre. We compared these across three groups as per their exposure to pediatric patients (routinely, occasionally or rarely exposed to pediatric patients).

Method Consensually validated questionnaire containing combination of questions from basic (must know) and advanced (nice to know) areas of knowledge about nursing pediatric patients and questions related to nurses' perception about pain in pediatric patients was administered to eligible nursing staff at Rural Tertiary Care Hospital in Western India. The responses were analyzed using descriptive statistics and comparisons were made by chi-square test. Result 351 usable questionnaires (83.37\%) out of 421 were returned. The knowledge of the nurses regarding pain was observed to be poor. $60 \%$ of all the nurses had complete knowledge of all the basic questions asked. Only $3.1 \%$ had answered all of the five advanced questions correctly, while $96.9 \%$ of the nurses had answered one or more questions incorrectly.

Conclusions The deficit in knowledge and shortcomings in perception needs to be addressed and steps need to be taken to improve the nurse's knowledge and modify beliefs and attitude of the nursing staff towards the pain of the pediatric patients.

\section{IMPROVING PAIN MANAGEMENT IN TERTIARY PEDIATRIC HOSPITAL- HIGH HOPES AND ORDINARY PROBLEMS}

doi:10.1136/archdischild-2012-302724.1626

J Kalbowiak, M Manowska, P Łaniewski- Wołk, E Pietraszek- Jezierska. Anaethesia and Intensive Care, Children's Memorial Health Institute, Warszawa, Poland

Background and Aims Observation of pain management problems in tertiary pediatric hospital (Children's Memorial Health Institute, Warsaw, Poland) resulted in anaesthesia and intensive care team initiative of pain practice improvement.

Methods In November 2011 Pain Treatment Committee represented by 22 doctors and 20 nurses of all hospital departments was established.

In November/December 2011 pilot training programme for nurses of Pediatric Urology, Neurosurgery and Cardiac Surgery Departments was performed.
In December 2011 written pain assessment and treatment guidelines accepted by the committee members and hospital administration where announced. They included:

- obligatory pain intensity assessment with age/communication skills- appropriate tools (NIPS, FLACC, Wong- Baker, VAS).

- pain treatment adequate to individual pain intensity with multimodal analgesia use.

- restriction in muscular injections of analgesics.

- analgesics dosing guidelines.

- perioperative analgesia algorithms based on predicted pain intensity.

Between February and March 2012201 practitioners and 391 nurses participated in pain assessment and management seminars.

Results Preliminary report on analgesia practice in surgical units revealed:

- poor compliance with pain assessment guidelines

- $100 \%$ reduction in intramuscular opioid use in one of the departments.

- improvement in analgesic prescriptions practice with individual variability between practitioners.

- increase in number of pain consultations

- no improvement with use of regional analgesia, insufficient number of PCA pumps.

Conclusions During few months after introducing hospital pain management guidelines we notice a change in pain practice but many problems still exist. Analgesia quality improvement is longterm process requiring multidisciplinary approach.

\section{APPLICATION OF PULSE OXIMETRY FOR DETERMINING THE STATUS OF THE TOOTH IN CHILDREN}

doi:10.1136/archdischild-2012-302724.1627

H Jafarzadeh, N Dastmalchi. Mashhad University of Medical Sciences, Mashhad, Iran

Tests relying on the passage of the light through a tooth have been considered to be a suitable means of assessing pulp vitality in children. Pulse oximetry which is an effective, objective oxygen saturation monitoring technique broadly used in medicine for recording blood oxygen saturation levels, can also be used in endodontic diagnosis for differential diagnosis of vital and necrotic pulps in young teeth. In this system, light is passed from a photoelectric diode across the tooth structure into a receptor. The instrument detects changes in absorption in both red and infrared light caused by alteration in tissue volume during the cardiac cycle. However, there are some limitations such as the effect of increased acidity and metabolic rate which cause deoxygenating of hemoglobin and changes in the blood oxygen saturation. Because this test produces no noxious stimuli, children usually accept it more readily than routine methods. In this lecture, principles, indications, limitations, influencing factors, and variations in probe design for dental usage in children would be presented.

\section{OXYGEN PRESCRIBING AND SATURATION TARGETING IN NEONATAL INTENSIVE CARE}

doi:10.1136/archdischild-2012-302724.1628

R Cronin, J Behrsin, P Bustani. Neonatal Unit, Jessop Wing, Sheffield, UK

Background Many newborns require oxygen; this should be prescribed as with other medical gases. In babies due to the risks from both hypoxia and hyperoxia oxygen saturation targeting to provide optimal safe oxygenation is commonly utilized.

Guidelines for the appropriate target saturations and hence monitoring alarm limits have changed locally in light of evidence from the SUPPORT ${ }^{1}$ and BOOST II ${ }^{2}$ trials. 